\title{
Are Household Food Expenditures Responsive to Entry Into the Supplemental Nutrition Assistance Program?*
}

\author{
Jiyoon (June) Kim, University of Michigan \\ H. Luke Shaefer, University of Michigan
}

Objective. This study examines changes in household food expenditures in the months directly around entry into the Supplemental Nutrition Assistance Program (SNAP). Methods. Using monthly data on SNAP participation from the Panel Study of Income Dynamics (PSID), we estimate food-expenditures-to-food-needs ratio with an event-study specification. Results. Upon entering the program, a substantially lower share of out-of-pocket spending accounts for SNAP households' food budgets. However, these expenditures are largely replaced with SNAP benefits, resulting in little changes in total food expenditures. We also identify the co-occurrence of household economic shocks at the point of SNAP entry. Conclusion. SNAP entry is preceded by reduced rates of employment and marriage among heads of households, suggesting that household economic shocks may trigger SNAP entry. We find that SNAP benefits visibly appear to act as an important safety net for households who have recently entered the program, cushioning them from the extent of a negative shock in food expenditures that they might otherwise have faced.

The Supplemental Nutrition Assistance Program (SNAP) is the federal government's largest nutrition assistance program. SNAP provides participating low-income families a monthly benefit in the form of Electronic Benefit Transfers (EBT) that can only be used to purchase food at participating retailers. During fiscal year 2013, SNAP spending was a record $\$ 82.5$ billion, with an average caseload of 47.7 million people per month, about one of every seven U.S. residents.

Because of growing size of the program and unprecedented number of recipients in SNAP, there has been substantial policy interest in evaluating the effects of the program on individual and family outcomes. Among a wide range of outcomes, SNAP's effect on households' food security has been highlighted in the literature. However, self-selection complicates efforts to evaluate the relationship between SNAP receipt and food insecurity because it is likely that households most in need of assistance are also the most likely to apply for benefits. In fact, Gundersen and Kreider (2008), and other studies have shown that low-income households that receive SNAP are more likely to report food insecurity than similar nonparticipating households (see Bartfeld and Dunifon, 2006; Gibson-Davis and Foster, 2006; Gundersen and Kreider, 2008; Jensen, 2002; Wilde, 2007; Nord and Wilde, 2005). In an attempt to address this issue, some recent studies use more sophisticated

\footnotetext{
*Direct correspondence to Jiyoon (June) Kim, Department of Economics, University of Michigan, 611 Tappan Avenue, Ann Arbor, MI 48109 〈junekim@umich.edu〉. The author will share all data and coding for replication purposes. The authors would like to thank Sheldon Danziger, Mark Nord, Laura Tiehen, Charlie Brown, Patrick Wightman, and Bridget Lavelle for insightful ideas, comments, and suggestions.
} 
techniques, including instrumental variable approaches, and find a negative relationship between SNAP participation and food insecurity (Nord and Golla, 2009; Mykerezi and Mills, 2010; Ratcliffe and McKernan, 2011; Gutierrez and Shaefer, 2013). Yet much remains to be learned about the relationship between SNAP and food insecurity.

The mechanism by which SNAP would reduce food insecurity is through expanding recipient households' food budgets. Accordingly, direct evidence of SNAP's ameliorative effect should be apparent in the food expenditures of these households, pre- and postSNAP entry. Do food expenditures rise, fall, or remain stable in the months following program entry? Do SNAP dollars replace or supplement out-of-pocket spending? What other changes happen to a family around the time of SNAP entry? We delve into these questions by adopting an event-study framework (Jacobson et al., 1993) to draw monthto-month trends in food expenditures before and after SNAP entry.

We use the Panel Study of Income Dynamics (PSID), the only panel data set with measures of both household food expenditures and SNAP participation by month. Taking advantage of the PSID's panel structure, we create an event-study analysis sample identified by the month relative to the SNAP entry. We then use these observations to track trends in household food expenditures for the 12 months prior to SNAP entry, and the 12 months following SNAP entry. Our findings suggest that SNAP shields new entrants from a substantial fall in food expenditures. Even after controlling for demographic characteristics as well as time and state fixed effects, our event-study estimation reveals that total food expenditures show a stable and smooth trend in the months surrounding SNAP entry. This is found to be largely due to SNAP replacing a great share of households' food budget after program entry. We also demonstrate that there are household shocks that co-occur with SNAP entry, such as new spells of unemployment or marital dissolution, suggesting that these shocks may trigger SNAP entry.

\section{Background and Literature Review}

With an average monthly benefit of $\$ 159$ per person and a maximum monthly benefit of $\$ 668$ for a family of four, SNAP benefits increase the gross incomes of participating households by 39 percent, and by 45 percent for households with children (Dahl et al., 2012). Therefore, we might expect that SNAP would increase the food expenditures or overall purchasing power of participating households, and that this should reduce their food insecurity.

Using a novel approach, Nord and Golla (2009) find evidence that suggests the food security of households deteriorates considerably in the few months prior to SNAP entry. They match households across two annual Current Population Survey Food Security Supplements (CPS-FSS). They exploit food security measures, combined with monthly data on SNAP receipt, to identify two samples: (1) households that entered SNAP during the 12 months before the CPS-FSS's food security outcome is measured and (2) households that entered SNAP during the 12 months after they completed the survey. They find that household food insecurity increases during the few months prior to SNAP entry, and decreases in the months following.

This improved food security after SNAP entry could mainly work through expanded resources available through SNAP dollars. Therefore, understanding the effects of SNAP on household food expenditures would bolster possible mechanisms for ameliorative effects of SNAP that are evident in broad literatures. SNAP benefits should improve food security through increased purchasing power for households' food expenditures. A number of papers, mostly using data from 20 years ago or prior, have examined the impact of SNAP 
on household food expenditures, focusing on how much SNAP benefits increase household food expenditures compared to what would have occurred with an equal amount of cash. In a review of this literature, Fox, Hamilton, and Lin (2004) note that 32 studies utilized one of three research approaches: (1) participant versus nonparticipant comparisons, (2) dose-response estimates, and (3) cash-out demonstrations that provide an experimental group with food stamps and a control group with an equivalent cash benefit. These studies consistently find that food stamp participants spend more on food than do comparable nonparticipants.

Fraker (1990) reports on a major cash-out randomized trial that finds that the marginal propensity to consume food out of food stamps is two to ten times higher than the estimated marginal propensity to consume food out of cash income. A more recent study by Hoynes and Schanzenbach (2007) exploits variation in the timing of the implementation of the Food Stamps Program (1963-1975) across counties. They find that the introduction of the program led to an overall increase in household total food expenditures among recipients, a decreased propensity to eat out, and mixed results for cash food expenditures (out-of-pocket expenditures).

Yet, none of these studies dynamically examine how food-expenditure patterns of households on SNAP change in the months before and after SNAP entry. The current article employs an event-study methodology that follows month-to-month trends in food expenditures and provides further evidence of the dynamic relationship between SNAP entry and food expenditures.

We may conjecture that trends in food expenditures follow that of food security, given that food expenditures and food security generally move in the same direction. Aligned with findings by Nord and Golla (2009) that food insecurity increased during the few months prior to SNAP entry, household food expenditures may also fall prior to SNAP entry, particularly if economic shocks, such as job loss or marital dissolution, are primary precipitators of new applications for benefits. Mabli and Ohls (2012:5) say that "trigger events" reflect "a series of complex lag structures in both the eligibility and participation equations." New entrants not previously receiving benefits may experience some changes in their circumstances that lead them to apply. Indeed, a number of studies find that SNAP is responsive to economic shocks such as job loss or marital dissolution (Mabli and Ohls, 2012; Hernandez and Ziol-Guest, 2009). Thus, such shocks may be a primary cause for falling household food expenditures among new SNAP entrants.

Do households' food-expenditure levels fluctuate prior to entering SNAP and recover afterward? Does SNAP help households maintain constant food expenditures levels, replacing the household's own expenditures on food with public funding during times of need? To provide answers to these questions, we simulate household food expenditures in the 12 months leading up to SNAP entry, and in the 12-month period after households enter the SNAP program, using a nationally representative sample.

\section{Data and Measures}

The PSID is one of the world's longest-running household panel surveys. It is among the most important surveys for social science research and evaluation of government policies. It is the main source for information on changes in income and poverty over time, allowing longitudinal analysis of income and consumption. Available waves were collected annually from 1968 through 1997, and then biennially after 1997 up until 2011.

The PSID is uniquely suited for our analysis because it is the only nationally representative panel data set with both measures of household food expenditures and monthly SNAP 
program participation. We pool data from the four most recent waves of the PSID: 2001, 2003, 2005, and 2007. ${ }^{1}$ In each wave, households are asked about average food expenditures at the time of the interview, ${ }^{2}$ and monthly SNAP participation for the previous two calendar years and the current year. For instance, in the 2001 wave, respondents report average food expenditures at the time of the interview, as well as month-by-month SNAP participation records from January 1999 through the interview month of 2001.

Underreporting of public transfer receipt in the PSID remains a limitation (Gundersen and Kreider, 2008). Meyer, Mok, and Sullivan (2009) provide estimates of the extent of underreporting for 10 transfer programs in five major nationally representative surveys, and find that approximately 80 percent of food stamp dollars are reported in the PSID and SIPP, while in the other surveys it is closer to 60 percent. For years beginning with 2003, a significant improvement is visibly detected in the PSID, with a higher reporting rate than that of the SIPP.

Food expenditures are reported in three different categories in the PSID_food at home, food delivered, and food eaten out. If a household is currently a SNAP recipient, a separate question about the amount of the SNAP benefit is asked. ${ }^{3}$ Accordingly, we construct total food expenditures by summing (1) out-of-pocket spending on food at home (i.e., spending not using SNAP benefits), (2) food delivered, (3) food eaten out, and (4) SNAP benefit amount. Li et al. (2010) benchmark the quality of the PSID expenditures data by comparing them with data from the Consumer Expenditures Survey (CE). They find that the PSID expenditures compare favorably with those in the CE data, with spending amounts closely aligning in every expenditure category.

The PSID simulates the USDA's food needs standard for each household-an estimate of expected weekly food costs based on the Low-Cost Food Plan ${ }^{4}$ for a family of the same size and composition. The food needs standard is widely used in order to account for family composition when measuring food expenditures (Zeldes, 1989; Stephen, 2004). The PSID food needs variable, however, is measured in reference to the previous year. Because of different accounting periods of food expenditures and food needs, the food needs variable should not be used at face value when calculating the ratio between the two. Therefore, we reconstructed the food needs variable with family composition information as of the interview date, using the standard formula taken from USDA Low-Cost Food Plan (Appendix B).

Our main outcome variable is, therefore, the ratio between the food expenditures of the household, over that family's USDA food needs standard, both of which are measured at the time of interview. By dividing a household's food expenditures by its food needs threshold, we account for differences in the family size and composition of heterogeneous households. Moreover, the ratio is easy to interpret-if the ratio is greater than 1, a household is spending

\footnotetext{
${ }^{1}$ We do not use the 2009 and 2011 waves because monthly SNAP participation questions were omitted from the 2009 wave.

${ }^{2}$ Respondents are asked to report the amount they currently spend in an average week, but they are allowed to report annual or monthly amounts. We adjust the reported amount to be representative of monthly food expenditures to be consistent with monthly measures of SNAP participation.

${ }^{3}$ See Appendix A for the question wording.

${ }^{4}$ The USDA provides guides for selecting nutritious diets at home at different cost levels Thrifty

Low

Moderate

Liberal
}

The Thrifty Plan, which is the least costly of the four, is the basis for the SNAP benefits. The Low and Moderate programs provide appropriate diets for most people. The Liberal Plan, which doubles the cost of the Thrifty Plan, permits a greater variety than the other plans. We use the built-in variable in the PSID, food needs standard based on the Low-Cost Food Plan, for the analysis. 
more than the national average needed for food for the Low-Cost Food Plan by a household with its composition. If the ratio is less than 1 , vice versa.

\section{Methodology and Identification Strategy}

\section{Construction of Analysis Sample}

We use food-expenditure measures, combined with monthly data on SNAP receipt, to identify two samples: (1) households that entered SNAP during the 12 months before food-expenditure outcome is measured (food expenditures are treated by SNAP) and (2) households that entered SNAP during the 12 months after they completed the survey (food expenditures are not treated by SNAP).

This sample construction, however, could be complicated by the fact that a family's monthly SNAP participation data are coming from two consecutive PSID waves that are two years apart. For example, if a household was interviewed in April 2001 for the 2001 PSID wave, monthly data on SNAP receipt for 2000 and early 2001 are obtained from the same wave. However, monthly data on SNAP receipt for later 2001 and 2002 are obtained from 2003 PSID wave.

We should note that not all families stay the same over the PSID waves. Some drop from the sample because of attrition. ${ }^{5}$ Others change family composition between waves. For example, families can split into two or more subfamilies because of marriage or divorce. In order to avoid the results being confounded by nonrandom family composition change across waves, we first restrict to households that do not split into multiple families across two waves. This guarantees that monthly SNAP receipt information from two sequential PSID waves corresponds to the same family. It is reassuring that the proportion of families that stayed the same with respect to their composition is relatively high across waves: 91 percent (2001-2003), 85 percent (2003-2005), 87.5 percent (2005-2007), and 85.6 percent (2007-2009).

The second restriction that we impose on our sample is that we focus on households that were not on SNAP for at least 12 months prior to the observed SNAP entry. This restriction provides an estimate of food expenditures without contamination by households that cycle on and off of SNAP. Because we are interested in SNAP entry rather than SNAP participation, this restriction leads us to exclude families who use SNAP over long durations. We do not impose any restriction with respect to the duration of a family's SNAP spell, once it starts—some households may have received SNAP for only one month, whereas others may have received benefits for 12 months or more. ${ }^{6}$

To understand the effects of the two sample restrictions, we conducted a balance test of demographic variables between households included in our final analysis and households that are excluded due to the two sample restrictions. Table 1 presents the result of the test. Our final sample includes almost a third ${ }^{7}$ of all families ever reporting SNAP in the PSID waves. In some dimensions such as employment status and race, our final analysis sample is slightly more positively selected, but not considerably so. This is not a surprise since we limit to those who have no SNAP history for at least a year prior to the observed entry,

\footnotetext{
${ }^{5}$ The proportion of families who drop between waves is the following: 4.9 percent (2001-2003), 6.8 percent (2003-2005), and 4.9 percent (2005-2007).

${ }^{6}$ In sensitivity analyses, we restrict the sample to households whose SNAP spells are at least six months or longer. Results remain substantively similar.

About 60 percent of ever-receiving SNAP households are excluded due to a 12 -month no-entry restriction, and an additional 7 percent are excluded due to a no-family-composition-change restriction.
} 
TABLE 1

Comparison of Demographic Characteristics Between Families in Final Sample and Those Excluded

\begin{tabular}{lcccc}
\hline Demographic Variables & In Sample & Excluded & Difference & T-Statistics \\
\hline Age of head & 37.5 & 39.8 & -2.3 & -5.3 \\
Head is male & 0.53 & 0.45 & 0.07 & 4.6 \\
Number of children & 1.3 & 1.6 & -0.3 & -7.2 \\
Head is married & 0.27 & 0.26 & 0.01 & 0.98 \\
Own home & 0.31 & 0.31 & 0 & 0.07 \\
Head working & 0.6 & 0.55 & 0.05 & 3.5 \\
Head white & 0.33 & 0.29 & 0.04 & 2.7 \\
Head black & 0.59 & 0.64 & -0.04 & -2.8 \\
Head's education & 11.6 & 11.4 & 0.18 & 2.2 \\
Observations & 1,477 & 2,989 & & \\
\hline
\end{tabular}

NoTE: Two sample restrictions, (1) 12-month no SNAP entry and (2) no family split across two consecutive PSID waves, result in some observations excluded from the analysis sample (2,989 observations). In Table 1, we implement a balance test between our analysis sample (1,477 observations) and those who are excluded (2,989 observations).

SOURCE: Authors' analysis of the PSID, 2001, 2003, 2005, and 2007 waves.

which excludes static SNAP participants, who are more disadvantaged with respect to some observable characteristics. In terms of marital status, home ownership, and education, both groups look substantially similar to each other. Thus, our findings are based on estimates from a sample of relatively new SNAP entrants and may not reflect long-term static SNAP participants. Still, our sample consists of nearly a third of those ever reporting SNAP receipt in the PSID for the years under study. Given these results, it is likely that this study provides lower bounds of the food-expenditure pattern, suggesting that the patterns may be more drastic when all SNAP recipients are taken into account.

After the two restrictions imposed, finally, we group households according to the months between SNAP entry and the measurement of food expenditures. Households that entered SNAP 12 months before reporting food expenditures are grouped together; those entering SNAP 11 months before reporting food expenditures are grouped together; continuing until the final group consists of households entering SNAP 12 months after the food expenditures are measured. Thus, the study sample represents a progression of independent families sorted on the timing of the observation when they report food expenditures relative to the month of SNAP entry.

Our unit of analysis is the household-SNAP spells. As long as the conditions discussed above are met, households with multiple SNAP spells over the course of the four PSID waves can be included multiple times. The primary analysis sample consists of the 1,764 household-SNAP spells from 1,477 unique households.

\section{Analytic Methods: Event-Study Analysis Before and After SNAP Entry}

To explore the underlying trends, we simply plot the average food-expenditures-to-foodneeds ratio across months surrounding SNAP entry in Figure 1 as our descriptive analysis. We evaluate our descriptive trend in an event-study specification to test the statistical significance of the association between food-expenditures-to-food-needs ratio and months 


\section{FIGURE 1}

Descriptive Trend: Alean Ratio of Food Expenditures to Food Needs by Time to SNAP Entry

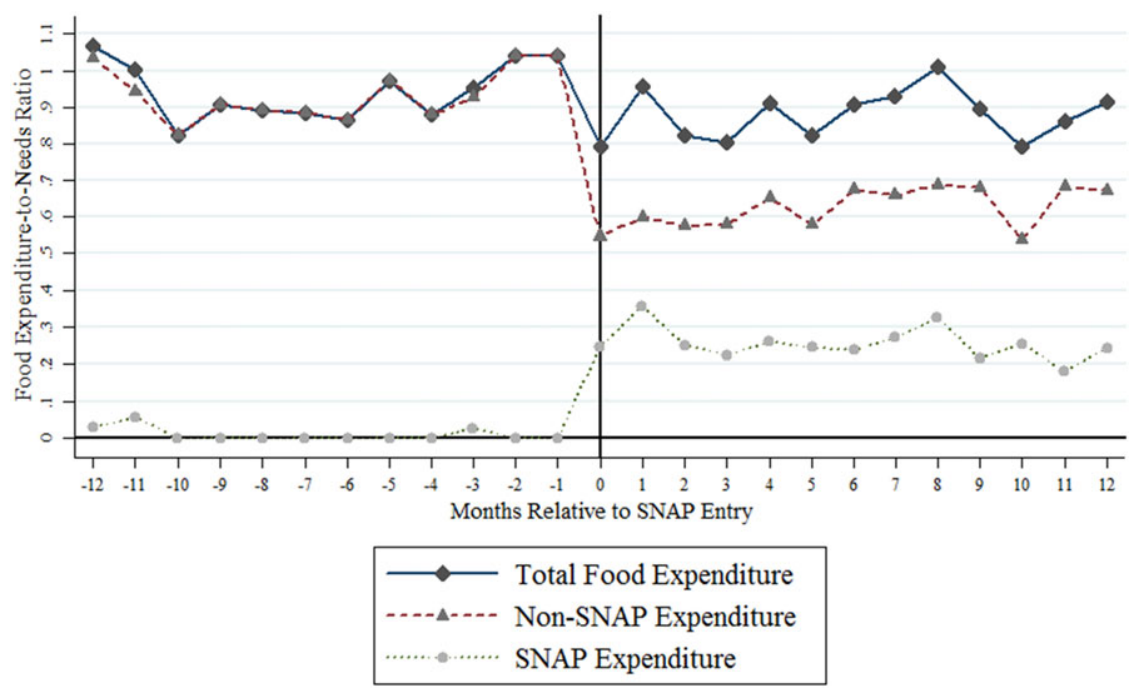

NOTE: Estimates are the mean ratio of household food expenditures to food needs, broken down into SNAP and non-SNAP expenditures. It plots a mean outcome at each time point, unadjusted for controls.

SOURCE: Authors' analysis of the PSID, 2001, 2003, 2005, and 2007 waves.

relative to SNAP entry. Specifically, Equation (1) shows the event-study model:

$$
\begin{aligned}
\left(\frac{\text { Food Expenditures }}{\text { Food Needs }}\right)_{\mathrm{ht}}= & \beta_{0}+\sum_{\mathrm{i}=-12}^{12} \beta_{\mathrm{i}} \cdot I\left(\tau_{\mathrm{ht}}=i\right)+\pi \cdot X_{\mathrm{ht}}+\rho_{\mathrm{t}}+\theta_{y}+\sigma_{s} \\
& +\sigma_{\mathrm{s}} \cdot y+\varepsilon_{\mathrm{ht}} .
\end{aligned}
$$

$\tau_{\mathrm{ht}}$ denotes the number of months relative to the event of SNAP entry for household $h$ interviewed in month $t$. It is defined so that $\tau_{\mathrm{ht}}=0$ if food expenditures are measured in the same month as the SNAP entry. For $\tau_{\mathrm{ht}}>0$, food expenditures are treated by SNAP. For instance, $\tau_{\mathrm{ht}}=1$ indicates food expenditures measured one month after SNAP entry. For $\tau_{\mathrm{ht}}<0$, food expenditures are untreated by SNAP (i.e., food expenditures are measured before the program starts). When drawing plots, we omit the $\tau_{\mathrm{ht}}=0$ category as a reference group so that the $\beta_{\mathrm{i}}$ coefficients map out differences relative to the outcome at the time of SNAP entry. Our event-study model includes fixed effects for state $\left(\sigma_{s}\right)$, interview year $\left(\theta_{y}\right)$, and interview month $\left(\rho_{\mathrm{t}}\right)$ as well as state-specific time trends $\left(\sigma_{s} \cdot y\right)$. Given that the study period overlaps with a period of rapid changes in the SNAP as well as other welfare programs, this set of variables is to absorb any state-specific or time-specific trend that could affect food expenditures differently. Moreover, it captures heterogeneity in SNAP spells as well as food expenditures that could be taking place at different points of 
time over the study period. Demographic characteristics of household heads ${ }^{8}$ such as race, education, sex, marital status, and employment status ${ }^{9}$ are also controlled for.

In practice, demographic characteristics could be unbalanced in event-time in a way that interferes with identification. Therefore, we also run the event-study analysis with several fixed characteristics of heads as dependent variables. We show there is no significant trend in such characteristics as race, educational attainment, and age of head across months around SNAP entry. Job loss and marital dissolution are two major forms of household economic shock, which may trigger SNAP entry and thus may be associated with changes in household food expenditures. With this in mind, we also report an event-study analysis in which outcomes are not food expenditures, but are (1) whether a head of household is working and (2) whether a head of household is married.

\section{Findings}

\section{Descriptive Result}

Figure 1 simply plots the average ratio of household food expenditures to food needs for our analysis sample in reference to the event of SNAP entry (denoted as 0 on the horizontal axis). Months prior to SNAP entry begin with -12 and progress to -1 . Months following SNAP entry progress from +1 to +12 . The solid line is total food expenditures (non-SNAP food expenditures plus SNAP benefit amount) as a ratio to food needs. Total food expenditures can be decomposed into two components. The dashed line is non-SNAP food expenditures, which are the sum of out-of-pocket spending on food at home, food delivered, and food eaten out. The dotted line indicates the size of SNAP benefits as a ratio to food needs.

Examining first the non-SNAP food-expenditures-to-food-needs ratio trend line, we see stability during the months prior to SNAP entry, with some slight fluctuations. Households a year away from SNAP entry have a ratio more than 1, suggesting that their food expenditures exceed their USDA low-cost food needs standard. The ratio holds substantively steady between 0.82 and 1.04 .

The ratio of non-SNAP food expenditures to needs is substantially lower after SNAP entry. It is 0.55 during the month of SNAP entry, and the ratio bottoms out following SNAP entry at around 0.57 . This estimate indicates that households in the first month of SNAP participation have non-SNAP food expenditures that are a little over half of their household's basic food needs, substantially lower than households who are a few months away from entering SNAP. This pattern clearly reveals that SNAP recipients reduce their own out-of-pocket spending on food by replacing it with SNAP benefit.

Lastly, the lowest dotted line plots the introduction of SNAP resources into the household's food expenditures, rising from $0,{ }^{10}$ to accounting for an average of 0.36 of the

\footnotetext{
${ }^{8}$ The PSID defines household head as the person with the most financial responsibility and at least 16 years old. If this person is female and she has a husband in the family unit, then he is designated as "head." However, if the husband or boyfriend is incapacitated and unable to fulfill the functions of head, then the family unit will have a female head.

${ }_{9}^{9}$ Demographic controls include a set of indicators for a head being white, male, married, working, high school graduate, some college or more, and whether he or she owns a home.

${ }^{10}$ Even though we limit to the households that were not on SNAP for at least 12 months prior to the observed SNAP entry, there are some positive SNAP expenditures plotted in the months prior to SNAP entry in Figure 1. This is mainly due to reporting errors in the PSID: a negligible number of households reported positive SNAP dollar amounts even if they said they did not receive any SNAP benefits.
} 
household's basic food needs a month after. This proportion seems reasonable, given the heterogeneity of our sample employed in this study, which includes very different household configurations that would be eligible for greater or smaller SNAP benefits. Note also that some households included in our post-SNAP sample may have cycled off SNAP during the months following entry.

The effect of SNAP benefits on food expenditures is made clear by the wedge that opens up between the total food expenditures and the non-SNAP expenditures. While the non-SNAP food-expenditures-to-food-needs ratio is different considerably after the entry, overall food expenditures - which incorporate SNAP benefits - change far less, bottoming out at 0.80 for households three months following SNAP entry. When SNAP dollars are accounted for, the ratio of total food expenditures to food needs rises somewhat among households as they are more months past SNAP entry, so that by eight months following SNAP entry, the ratio is well within the range of pre-SNAP food expenditures. Even then, though, SNAP accounts for a considerable amount of the food expenditures of the average households. In essence, the SNAP benefits visibly appear to act as a "safety net" for households that have recently entered the program, cushioning them from the extent of a possible negative shock in food expenditures that they might otherwise have faced.

\section{Event Study}

Food-Expenditures-to-Food-Needs Ratio. Next, we test the relationship between our key outcome, the ratio of food expenditures to food needs, and months relative to SNAP entry using a multivariate framework. We estimate using the event-study methodology in an effort to control for various demographic characteristics of household heads. We also include time and state fixed effects so that the analysis is not confounded by seasonal trends or particular state characteristics.

Figure 2 plots the regression coefficients $\beta_{i}$ from Equation (1), where $i$ ranges from -12 to +12 months, separately for three different food expenditure outcomes. Since the month of SNAP entry $(i=0)$ is omitted as the reference month, the $\beta_{i}$ coefficients represent the difference in food-expenditures-to-food-needs ratio in month $i$ relative to the level at the SNAP entry. For example, the coefficient 0.36 on non-SNAP expenditures a month prior to SNAP entry implies the following: non-SNAP food expenditures account for a higher share of the household's basic food needs in a month prior to SNAP entry than in the month of SNAP entry, by 36 percent. It should be noted that the observed decrease in non-SNAP food expenditures-to-needs ratio starts from two months prior to SNAP entry. Some would think there would have been a more gradual decline in non-SNAP and total food expenditures before SNAP entry unless the provision of benefits is so quick. Indeed, our finding is surprisingly consistent with the current SNAP application system: in almost all states, SNAP offices make a decision on approval of an applicant's eligibility within 30 days of application (or within seven days for applicants who qualify for "expedited" SNAP benefits). The fact that most state SNAP offices make a decision on an applicant's eligibility within 30 days bolsters the reasoning behind a quick drop in non-SNAP food expenditures within one to two months of entry.

This event-study plot affirms that the descriptive trend in Figure 1 is also statistically supported even after controlling for households' demographics, time trend, and state characteristics. The non-SNAP food-expenditures-to-food-needs ratio changes dramatically around the time of SNAP entry and levels off during the following months after SNAP program entry. The total food-expenditures-to-food-needs ratio also fluctuates across the months surrounding SNAP entry, but the change is much less than that of non-SNAP 
FIGURE 2

Changes in Food-Expenditures-to-Food-Needs Ratio by Time to SNAP Entry: Event-Study Coefficients

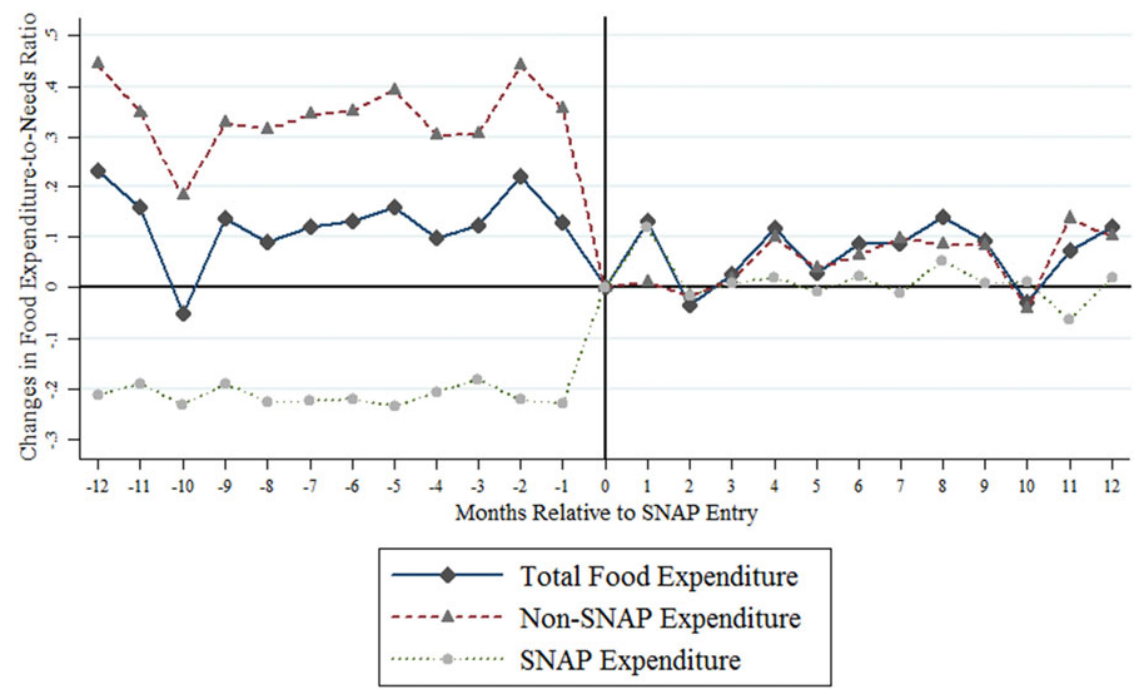

NOTE: It plots the regression coefficients $\beta_{i}$ from Equation (1) with the month of SNAP entry $(i=0)$ omitted as a reference group. The coefficient $\beta_{i}$ for each month represents the food-expenditures-to-food-needs ratio relative to the level at the month of SNAP entry, which is normalized to 0. Month, year, and state fixed effects as well as state-specific time trends are included. The regressions control for head's education, race, sex, marital and employment status, and whether the household owns a home.

SOURCE: Authors' analysis of the PSID, 2001, 2003, 2005, and 2007 waves.

expenditures. Overall, total food expenditures show a relatively stable and smooth trend across all event-times, suggesting the role of SNAP as a safety-net program for low-income households.

Co-Occurrence of Economic Shocks at the Time of SNAP Entry. Estimates presented in Figures 3 and 4 are consistent with previous research that finds that many households experience economic shocks prior to SNAP entry. Figure 3 plots the proportion of households in which the head is currently working. Note that the level of outcome is normalized to 0 at the time of entry; thus estimates in other event months are changes relative to the entry month. Before SNAP participation, the proportion of households with a working head is greater by 10 to 30 percentage points compared to the fraction at the time of SNAP entry. But it starts to lower substantially beginning two months prior to SNAP entry. We see a slow and gradual increase in the proportion of households with a working head in the post-SNAP sample.

A similar pattern is observed for marital status in Figure 4. Three months prior to SNAP entry, the proportion of married household heads is higher by 30 percentage points. The fraction of married heads hits the lowest point right before SNAP entry. High levels of marital dissolution just before program entry likely lead these families to seek governmental transfer thereafter. Both Figures 3 and 4 suggest that program entry is likely to be preceded by household economic shocks.

Some may be concerned about any possibility of unbalanced nature of pre- and postSNAP samples, which could possibly confound the results. In Appendix Figures 1-3, we 


\section{FIGURE 3}

\section{Proportion of Working Heads, with 95 Percent Confidence Intervals}

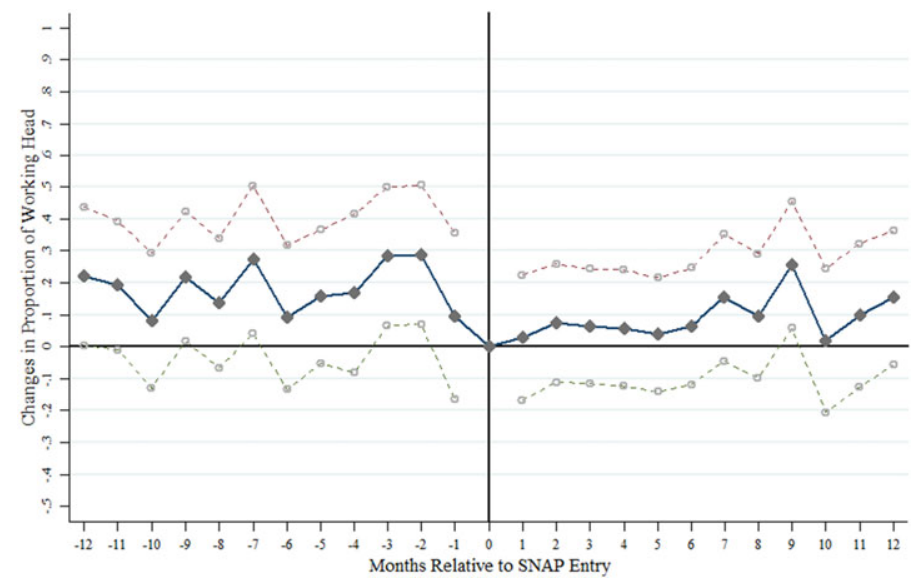

NOTE: It plots the regression coefficients $\beta_{i}$ from Equation (1) with the month of SNAP entry $(i=0)$ omitted as a reference group in the event-study model. Each data point represents the difference in the proportion of working heads relative to that at the month of SNAP entry, which is normalized to 0 . Month, year, and state fixed effects as well as state-specific time trends are included.

Source: Authors' analysis of the PSID, 2001, 2003, 2005, and 2007 waves.

\section{FIGURE 4}

\section{Proportion of Married Heads, with 95 Percent Confidence Intervals}

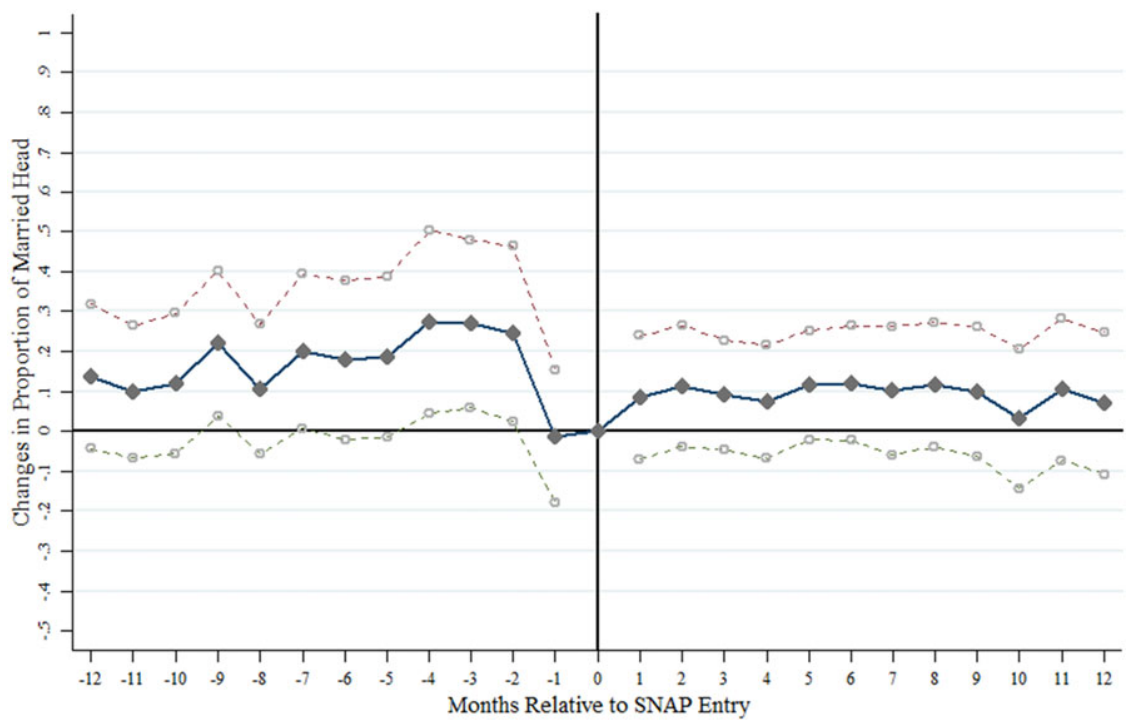

NOTE: It plots the regression coefficients $\beta_{i}$ from Equation (1) with the month of SNAP entry $(i=0)$ omitted as a reference group in the event-study model. Each data point represents the difference in the proportion of married heads relative to that at the month of SNAP entry, which is normalized to 0 . Month, year, and state fixed effects as well as state-specific time trends are included.

Source: Authors' analysis of the PSID, 2001, 2003, 2005, and 2007 waves. 
plot the similar plots but replace the outcomes with fixed characteristics of household heads: indicators for a head being black, years of schooling, and age of a head, respectively. The Appendix figures show that all estimates are not distinguishable from zero, with large standard errors for all months before and after SNAP entry. This reveals that there is no statistically significant underlying trend in any of these fixed characteristics across pre- and post-SNAP groups, giving us confidence that our findings are not driven by the nature of unbalanced characteristics of two samples.

\section{Conclusion}

Building upon a wide body of empirical literature that evaluates the impact of SNAP on economic, social, and health outcomes, this study provides direct evidence of SNAP, which is immediately reflected in household food expenditures. Taking advantage of the most recent PSID data available, with measures of both food expenditures and monthly SNAP participation, we depict the month-to-month food expenditures trend with an event-study analysis.

Our estimates uncover the dynamic relationship between food expenditures and SNAP entry. First, we start with descriptive analysis, simply plotting the average food-expendituresto-food-needs ratio for the 12 months leading up to SNAP entry to 12 months after SNAP entry. Then we confirm that this descriptive pattern is supported with statistical significance in the multivariate event-study analyses. Both descriptive and multivariate analyses consistently tell a similar story over the course of the two-year timeframe and identify a potentially ameliorative effect of SNAP. Specifically, we observe that estimated non-SNAP food expenditures become lower substantially at the time of SNAP entry, while changes in total food expenditures are buffered by increased expenditures using SNAP, suggesting a role of SNAP as a protection against economic shocks.

Furthermore, we replace the food-expenditure outcome with economic status of a household head to discover any changes happening to a family around the time of SNAP entry. We find a strong relationship between time to SNAP entrance and the proportion of households with working and married heads. This offers evidence that the timing of SNAP entry often coincides with the occurrence of household economic shocks.

In essence, the SNAP benefits visibly appear to act as a "safety net" for households that have recently entered the program, cushioning them from the extent of a negative shock in food expenditures that they might otherwise have faced. This study offers a credible estimate of to what extent SNAP may supplement participants' income and help them expand their food budget accordingly. Because of the program's large size and prevalence, there is substantial policy interest in evaluating its effectiveness. This study, thus, provides compelling evidence of SNAP's pivotal role in improving food expenditures for households that would otherwise be at greater risk of food insecurity and lack of food choices in the absence of this safety-net program. 


\section{Appendix A}

\section{Appendix FIGURE 1}

\section{Proportion of Black Heads, with 95 Percent Confidence Intervals}

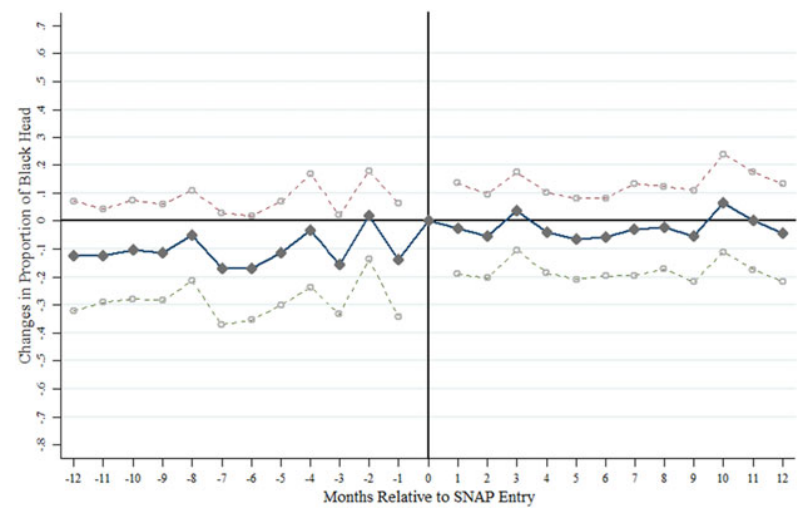

NOTE: It plots the regression coefficients $\beta_{i}$ from Equation (1) with the month of SNAP entry $(i=0)$ omitted as a reference group in the event-study model. Each data point represents the difference in the proportion of black heads relative to that at the month of SNAP entry, which is normalized to 0 . Month, year, and state fixed effects as well as state-specific time trends are included.

SOURCE: Authors' analysis of the PSID, 2001, 2003, 2005, and 2007 waves.

\section{Appendix FIGURE 2}

Heads' Years of Schooling, with 95 Percent Confidence Intervals

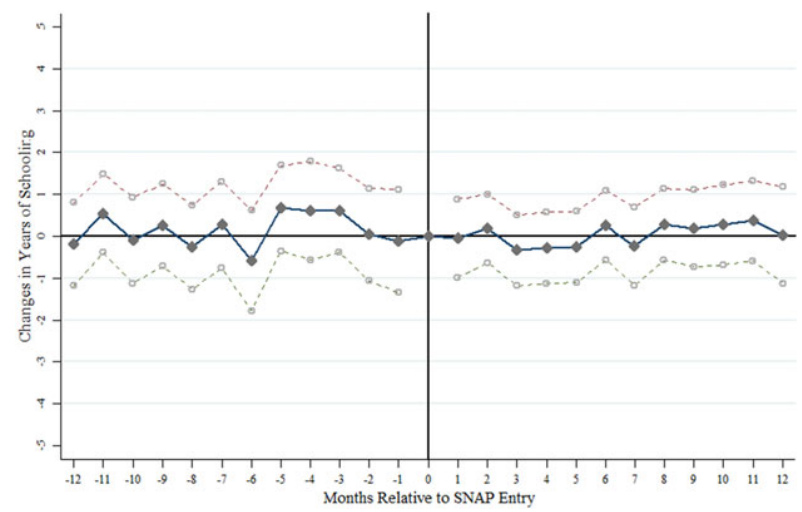

NOTE: It plots the regression coefficients $\beta_{i}$ from Equation (1) with the month of SNAP entry $(i=0)$ omitted as a reference group in the event-study model. Each data point represents the difference in years of schooling of heads relative to that at the month of SNAP entry, which is normalized to 0. Month, year, and state fixed effects as well as state-specific time trends are included.

SOURCE: Authors' analysis of the PSID, 2001, 2003, 2005, and 2007 waves. 


\section{Appendix FIGURE 3}

Age of Heads, with 95 Percent Confidence Intervals

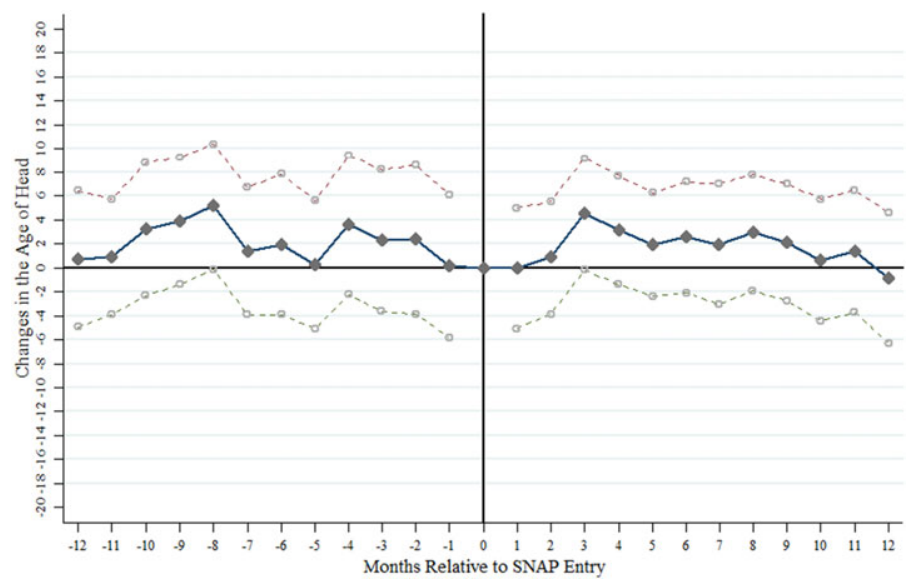

NOTE: It plots the regression coefficients $\beta_{i}$ from Equation (1) with the month of SNAP entry $(i=0)$ omitted as a reference group in the event-study model. Each data point represents the difference in age of heads relative to that at the month of SNAP entry, which is normalized to 0 . Month, year, and state fixed effects as well as state-specific time trends are included.

SOURCE: Authors' analysis of the PSID, 2001, 2003, 2005, and 2007 waves.

\section{SNAP Participation and Benefit Amount Questionnaire in PSID}

In the PSID interview, they ask about SNAP monthly participation and SNAP benefit amount of the previous two years and current year of the interview (e.g., in 2001 PSID, interviewers asked about 1999, 2000, and 2001 SNAP participation status). Here, we show the actual 2001 PSID questionnaire. The same questions were asked in 2003 through 2007 PSID.

Did you (or anyone in your family) use government food stamps at any time in (year)?

1. YES; $5 \mathrm{NO} ; 8 \mathrm{DK} ; 9 \mathrm{NA}$, refused

How many dollars' worth of stamps did you get in (year)?-AMOUNT

\begin{tabular}{ll}
\hline $0.01-999,996.99$ & Actual amount \\
$999,997.00$ & $\$ 999,997$ or more \\
$999,998.00$ & DK \\
$999,999.00$ & NA, refused \\
0.00 & Inap: did not receive food stamps in (year) \\
\hline
\end{tabular}

How many dollars' worth of stamps did you get in (year)?-TIME UNIT

3 Week

4 Two weeks

5 Month

6 Year

7 Other

$8 \mathrm{DK}$ 
9 NA, refused

0 Inap: did not receive food stamps in (year)

During which months did you receive food stamps?_JANUARY (year)

1 YES, 9 NA, DK

0 Inap: food stamps not used during this month; received no food stamps in (year)

During which months did you receive food stamps?-FEBRUARY (year)

During which months did you receive food stamps? - MARCH (year)

During which months did you receive food stamps?_-DECEMBER (year)

\section{Appendix B}

\section{How to Construct Annual USDA Needs Standard Variable}

1. Estimate the weekly food cost for each person according to age and sex at 1967 prices. These values are based on USDA Low-Cost Plan estimates of weekly food costs, then are summed for the family as it was at the time of the interview

Individual Food Standard (Low Cost)

$\$ 3.9$ for both males and females under age 4 years

$\$ 4.6$ for both males and females under age 4-6 years

$\$ 5.5$ for both males and females under age 7-9 years

$\$ 6.4$ for males aged $10-12$

$\$ 6.3$ for females aged $10-12$

$\$ 7.4$ for males aged $13-15$

$\$ 6.9$ for females aged $13-15$

$\$ 8.7$ for males aged $16-20$

$\$ 7.2$ for females aged $16-20$

$\$ 7.5$ for males aged $21-35$

$\$ 6.5$ for females aged $21-35$

$\$ 6.9$ for males aged $36-55$

$\$ 6.3$ for females aged $36-55$

$\$ 6.3$ for males aged 56 and older

$\$ 5.4$ for females aged 56 and older.

2 Multiply this by 52 to convert to an annual amount and further adjust for economies of scale by USDA rules as follows:

Single person: add 20 percent

Two persons: add 10 percent

Three persons: add 5 percent

Four persons: no change

Five persons: deduct 5 percent

Six or more persons: deduct 10 percent.

3 An additional adjustment for diseconomies of small households was made as follows:

4.89 times the food needs for single persons

3.70 times the food needs for two-person units

3.00 times the food needs for all other units. 


\section{REFERENCES}

Bartfeld, Judi, and Rachel Dunifon. 2006. "State-Level Predictors of Food Insecurity Among Households with Children." Journal of Policy Analysis and Management 25(4):921-42.

Dahl, Molly, Kathleen FitzGerald, Emily Holcombe, and Jonathan Schwabish. 2012. “The Supplemental Nutrition Assistance Program.” Congressional Budget Office Report. Washington, DC. Available at 〈http://www.cbo.gov/sites/default/files/04-19-SNAP.pdf).

Fox, Mary K., William Hamilton, and Biing-Hwan Lin. 2004. "Effects of Food Assistance and Nutrition Programs on Nutrition and Health: Volume 3 Literature Review." Food Assistance and Nutrition Research Report No. 19-3. Washington, DC: Economic Research Service, U.S. Department of Agriculture.

Fraker, T. M. 1990. The Effects of Food Stamps on Food Expenditures: A Review of the Literature. Alexandria, VA: Food and Nutrition Service, U.S. Department of Agriculture.

Gibson-Davis, C. M., and E. M. Foster. 2006. "A Cautionary Tale: Using Propensity Scores to Estimate the Effect of Food Stamps on Food Insecurity.” Social Service Review 80(1):93-126.

Gundersen, Craig, Dean Jolliffe, and Laura Tiehen. 2009. "The Challenge of Program Evaluation: When Increasing Program Participation Decreases the Relative Well-Being of Participants." Food Policy 34: 367-76.

Gundersen, Craig, and Brent Kreider. 2008. "Food Stamps and Food Insecurity: What Can be Learned in the Presence of Nonclassical Measurement Error?" Journal of Human Resources 43(2):35282.

Gutierrez, Italo A., and H. Luke Shaefer. 2013. "The Supplemental Nutrition Assistance Program and Material Hardships Among Low-Income Households with Children.” Social Service Review 87(4):75379.

Hernandez, Daphne C., and Kathleen M. Ziol-Guest. 2009. "Income Volatility and Family Structure Patterns: Associations Between Stability and Change in Food Stamp Program Participation." Journal of Family and Economic Issues 30(4):357-71.

Hoynes, Hilary W., and Diane Schanzenbach. 2007. "Consumption Responses to In-Kind Transfers: Evidence from the Introduction of the Food Stamps Program.” NBER Working Paper 13025. National Bureau of Economic Research.

Jacobson, Louis S., Robert J. Lalonde, and Daniel G. Sullivan. 1993. “Earnings Losses of Displaced Workers.” American Economic Review 83(4):685-709.

Jensen, Helen H. 2002. "Food Insecurity and the Food Stamps Program." American Journal of Agricultural Economics 84(5):1215-28.

Li, Geng, Robert F. Schoeni, Sheldon Danziger, and Kerwin Kofi Charles. 2010. "New Expenditures Data in the PSID: Comparisons with the CE.” Monthly Labor Review 133(2):29-39.

Mabli, James, and James C. Ohls. 2012. "Supplemental Nutrition Assistance Program Dynamics and Employment Transitions: The Role of Employment Instability.” Applied Economic Perspectives and Policy 34(1):187213.

Meyer, Bruce D., Wallace K. Mok, and James Xavier Sullivan. 2009. “The Under-Reporting of Transfers in Household Surveys: Its Nature and Consequences." NBER Working Papers 15181. National Bureau of Economic Research.

Mykerezi, Elton, and Bradford F. Mills. 2010. “The Impact of Food Stamp Program Participation on Household Food Insecurity.” American Journal of Agricultural Economics 92(5):1379-91.

Nord, Mark, and Marie Golla. 2009. "Does SNAP Decrease Food Insecurity? Untangling the Self-Selection Effect.” U.S. Department of Agriculture, ERS, Economic Research Report Number 85.

Nord, Mark, and Parke Wilde. 2005. "The Effects of Food Stamps on Food Security: A Panel Data Approach." Review of Agricultural Economics 27(3):425-32.

Ratcliffe, Caroline, and Signe-Mary McKernan. 2011. "How Much Does the Supplemental Nutrition Assistance Program Reduce Food Insecurity?” American Journal of Agricultural Economics 93(4):108298. 
Stephen, Melvin Jr. 2004. "Job Loss Expectations, Realizations and Household Consumption Behavior." Review of Economics and Statistics 86(1):253-69.

Wilde, Parke. 2007. "Measuring the Effect of Food Stamps on Food Insecurity and Hunger: Research and Policy Considerations.” Journal of Nutrition 137:307-10.

Zeldes, Steven P. 1989. "Consumption and Liquidity Constraints: An Empirical Investigation.” Journal of Political Economy 97(2):305-46. 